\title{
Navigation Mode Study of Mobile Application Based On Eye Tracking Technology
}

\author{
Fu Jiuqiang
}

Beijing Institute of Technology, Beijing, 100081, China

fujq0306@126.com

\begin{abstract}
Keywords: eye tracking technology, mobile application, navigation mode.
\end{abstract}
Abstract. Instances in this paper summarize six basic interface navigation modes of mobile application interfaces, analyze these six interface navigation modes' scenarios and features from information display and interactive operation, and most of the mobile phone interface structures base on and derive from them to a certain extent. In order to explore the visual browsing and searching rule of eye movement about different interface navigation modes, this study measured and recorded data by using eye tracking system. This paper adopted qualitative and quantitative research methods for searching visual browsing differences of interface navigation modes and the research result can be applied to design practice.

\section{Introduction}

For the limitation of mobile phone screen size, the screen content should be shown more simple and effective to users in the interface [1]. Because of this, designers are needed to organize information effectively and display the content through reasonable navigation mode to users. Now, designers and user interface researchers mainly focus on follows: the valid and reasonable layout of page information, how to guide people's visual behavior, discovering the characteristics of mobile application interface from the user's perspective and the cognition for improving the interface navigation. Excellent interface navigation can offer better browsing experience, which allow users to comprehend the interface structure quickly and predict the properties of the target interface, promoting the interface easier to use [2]. Eye tracking technology uses high-frequency camera to record data owning the advantages of high sampling frequency, high precision and small interference to users. Based on eye tracking technology, this paper explore users' recognition effect of page navigation modes, which can improve the objectivity and efficiency of user research.

There are many international researches about overall page navigation. The results of these studies can be used to guide Web page design and information layout, and provide vision parameters of human-computer interface design. But big-screen interface research results cannot directly applied to the small-screen interface. In the small-screen studies, researchers mainly study single element in the interface, less discuss the overall layout and navigation of the interface combining mobile platform features and eye tracking equipment. By analyzing the instances, this research aims to summarize six based navigation mode of mobile applications. Through visual browsing and eye movement analysis of different interface framework, research will explore the visual regularity of people's browsing screen, and try to apply these conclusions in practice.

\section{Six typical navigation modes of mobile application interface}

Vertical list mode. The content of mobile phone screen is often vertically displayed, but text is shown in the landscape normally, so the vertically arranged table can contain more information. The length of the list can have no limit, therefore users could view more contents by sliding up and down. Vertical list mode is often visually artistic and neat, so many users are ready to select it, which often be used in showing side elements, including directories, categories, content, as shown in Fig1 a.

Horizontal boxes mode. Horizontal box is a layout mode which can display element horizontally. Some common control, such as toolbar and Coverflow, just uses this mode. Restricted by the screen's 
width, this mode may display fewer content, but users can slide the screen or click on the arrows to view more content, which needs users to explore actively, as shown in Fig1 b.

Nine squares mode. Nine squares is a very classic layout mode, which owns simple appearance and is widely accepted by users. When the number of interface elements unchanged, using nine squares mode is appropriate. Although this mode sometimes gives the feeling of old ways, some variant of it becomes popular, such as the METRO style, as shown in Fig1 c.

TAB mode. TAB layout mode is to display coordinate information through horizontal or vertical tab. Compared with the traditional way of hierarchical structure, the element of TAB mode does not exceed 6, and only has a single level. When users click different options, the interface content updates, but the TAB area does not change, as shown in Fig1 d.

Multi-Layer boards mode. Multi-Layer boards layout mode commonly emerges in PAD terminals, and mobile phones relatively few use it. This mode is often used in traditional desktop software as navigation mode, looking much like a vertical titles in screen, which can display more information and be suitable for classification and displaying content, as shown in Fig1 e.

Sidebar mode. Sidebar is that page hides the contents first, and then expands as needed. This mode is more nature on the interactive experience and better integrates with original interface. Sidebar is often pulled from the left side of the screen, if it is pulled from the top or the bottom, we commonly call it as Message Manager Bar, as shown in Fig1 $\mathrm{f}$.

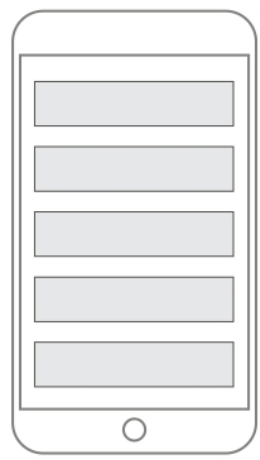

a)

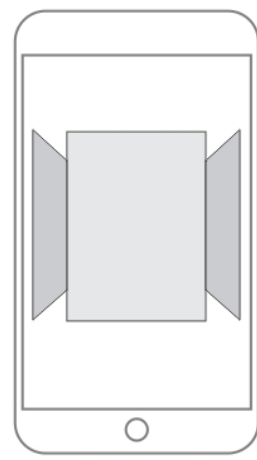

b)

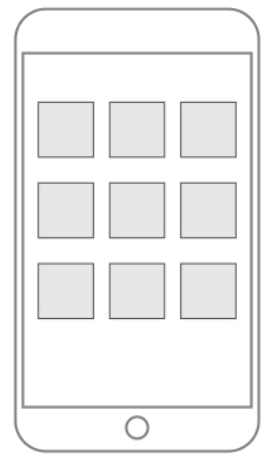

c)

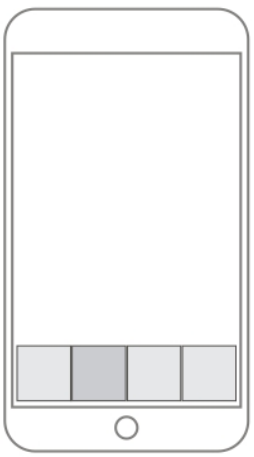

d)

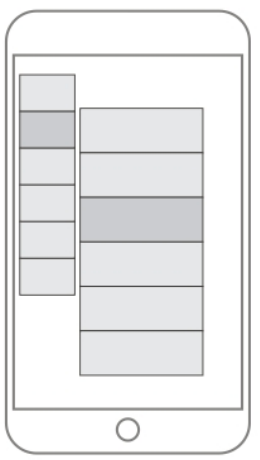

e)

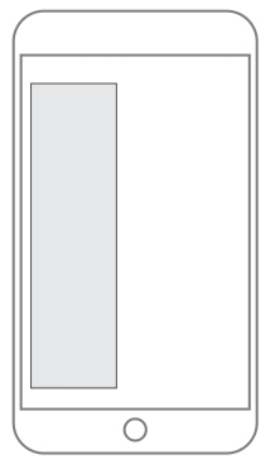

f)

Figure 1. Six typical navigation modes

\section{Experiment}

Previous step analyzed the scenario of 6 interface navigation modes from the information display and interactive operation. The next study will explore the different of visual browsing habits on different navigation modes and find the discrepancy of browsing efficiency. Previous studies have shown that eye tracking technology is an effective mean to study visual browsing in screen [3]. In eye tracking tests, experimenters often use eye tracking system to track participants for the search path of the visual interface, visual search changes because of visual interface, and search performance [4].

Experimental equipment, participants and tasks. Test equipment are an eye tracking system and smart phones (android and iOS). Test materials are 18 mobile application interfaces, and each navigation mode is divided into 3 interfaces. We will use Experiment Center, BeGaze software to record and analyze experimental data. The participants' eyes must be flush with the monitor Center with a distance of $60 \mathrm{~cm}$. Participants are 10 college students, have no behavior problems, are not familiar with the application interface of testing, and don't have trained behavior. In the experiment, participants are free to view 3 seconds and complete visual search tasks. A visual search task is that participants are going to search in an interface, and searching targets are primary navigation. When the participants find the target and click on it, the interface will automatically switch to the next screen after a successful search. The choices of participants will be recorded. Although the experiment uses a test 
computer and maybe have some discrepancy to the actual situation, such as users' response time, but for eye movement regular measurement, the results are still valid [5].

Experimental methods and design. The independent variables of this experiment are navigation modes of the interface type, according to the above classification of mobile navigation modes, application interface framework are divided into vertical list mode, horizontal boxes mode, nine squares mode, TAB mode, Multi-Layer boards mode and sidebar mode. Dependent variables in this experiment are five measurements of the eye movement test, which are fixation time $(\mathrm{T})$, fixation number $(\mathrm{N})$, the scanning path size $(\mathrm{S})$, target hit rate $(\mathrm{R})$, visual capture object $(\mathrm{O})$. In the above five measurements, the first three are direct indicators, which can directly access data by using eye tracking devices; the latter two are the indirect indicators, which need to be based on the original records of eye movement data.

Experimental procedures. Testers explained the testing process to participants, when the participants had correctly understood the operation, the test began. Experimental procedures were as follows: (1) Testers ran the program, connected eye tracking system, calibrated it, and opened the file that eye tracking system were needed to record. (2)Testers played interface graphics of mobile phones to participants and participants were free to browse interface for 3 seconds and then began a visual search task. Task subjects of the participants were that they should look for specified target in the mobile interface, when the target were found, clicked on the target. (3)The interface will automatically switched to the next interface after a successful search. The choices of participants were recorded, repeating the above operations, and the computer would automatically record participants' eye movement data. The same method was used to test the 10 subjects. The SPSS software was used to analyze data.

Experimental results. Experimental data. Figure 2 separately recorded ten users 'average fixation time, average fixation points and average scanning tracks length using the six interface navigation modes for completing the task. Analysis of variance in fixation time, fixation number and the scanning path size show that their p-values are less than 0.05 , which suggest that interface navigation modes on participants have a significant effect for fixation duration, number of fixation and the scanning path. According to the equation (1), data shows that the target hit rate of vertical list mode is $16.67 \%$, horizontal boxes mode is $12.31 \%$, nine squares mode is $29.54 \%$, TAB mode is $25.83 \%$, Multi-Layer boards mode is $18.76 \%$ and sidebar mode is $7.29 \%$.

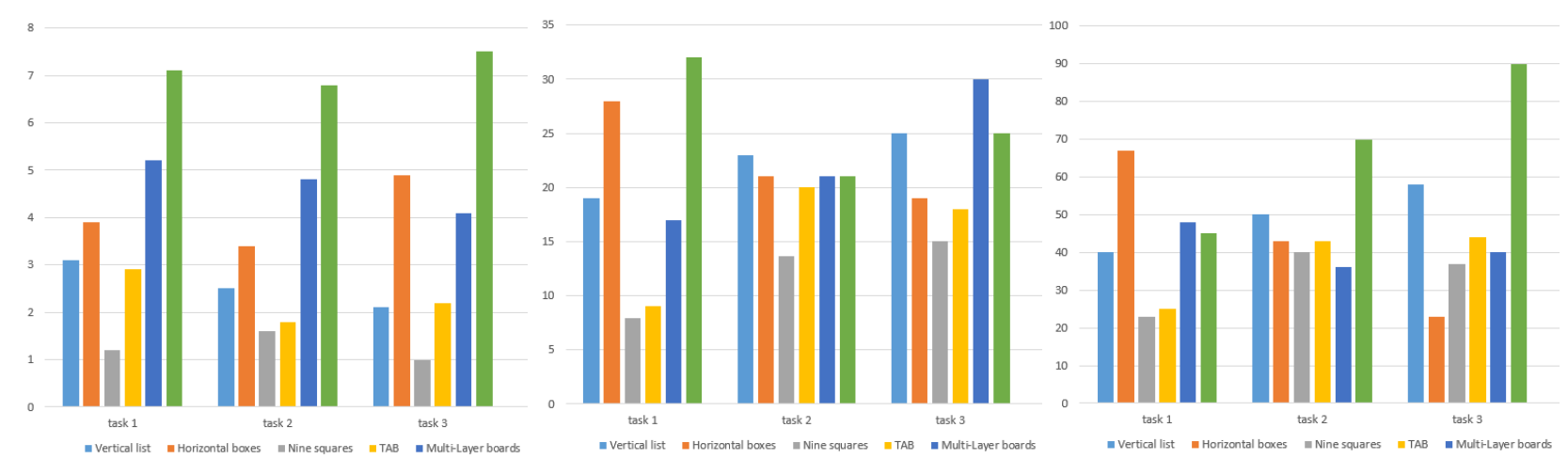

Fig2. Scanning path size, fixation time and fixation number

$$
\mathrm{R}=\left(\mathrm{N}_{1} / \mathrm{N}_{2}\right) \times 100 \%
$$

\section{Visual browsing principles}

Studies have shown that the more fixation time, the more difficult processing will be [6]. This test data shows: Nine squares mode has the shortest fixation time and scanning tracks length; Sidebar mode has the longest fixation time, more fixation points, and its scanning path is longer than any other modes of navigation, owning the lowest target hit rate. The efficiency and the level of difficulty in information 
processing of the other four models are in the middle. Studies on visual capture data find that experimental data display a contrary result of web interface. Through the analysis of eye tracing data, we can find, in the mobile phone interface, interface areas of the first fixation: About $59 \%$ visual capture object is located in the upper-left area of the mobile phone interface, and the effect of interface structure on visual capture object is not significant. About $73 \%$ eye movement trace is focus on the center, and rarely stay on fringe areas. The regional attention on the left side is ahead of the right zone, the fixation time on the left side is often longer than the right side. The navigation modes of Mobile phone interface has an obvious effect on the fixation point number and total search distance, but does not have a significant effect on visual capture object.

\section{Conclusions}

This study summarized 6 basic navigation modes about mobile phone interface, analyzed users' eye movement rules for different pages structure and found users' behavior discrepancy of using navigation through experiment. The results of this article has practical value for interface navigation design and can provide theoretical and data basis for designer, who can choose the most appropriate navigation modes for different needs.

\section{References}

[1] Sulaiman, S., Sohaimi, I.S.: An investigation to obtain a simple mobile phone interface for older adults. In: 2010 International Conference on Intelligent and Advanced Systems, pp. 1-4 (2010).

[2] Gajos, K.Z., Czerwinski, M., Tan, D.S., Weld, D.S.: Exploring the Design Space for Adaptive Graphical User Interfaces. In: Proc. 8th Int'l. Working Conf. Advanced Visual Interfaces (AVI 2006), pp. 201-208. ACM, New York (2006).

[3] Jacob, R.J.K., Karn, K.S.: Eye tracking in human-computer interaction and usability research: Ready to deliver the promises. In: Hyönä, J., Radach, R., Deubel, H. (eds.) The mind's Eye: Cognitive and Applied Aspects of Eye Movement Research, pp. 573-605. Elsevier, Amsterdam (2003).

[4] Xincan, Z., Hongfu, Z., Yongjun, R.: Overview of eye tracker and sight tracking technology. Comput. Eng. Appl. 42(12), 118-120 (2006).

[5] Sun, R.: Eye movement analysis technology and its application progress in aviation industry. J. Civil Aviat. Univ. China 21(4), 1-5 (2009).

[6] Granka, L., Joachims, T., Gay, G.: Eye-tracking analysis of user behavior in WWW search, Citeseer, pp. 25-29 (2004). 